\title{
Influence of as-built surface on fatigue strength and notch sensitivity of Ti6AI4V alloy produced by DMLS
}

\author{
Gianni Nicoletto ${ }^{1}$, Radomila Konečná ${ }^{2}$ Ludvík Kunz ${ }^{3}$, Martin Frkáň² \\ ${ }^{1}$ University of Parma, Parma, Italy \\ ${ }^{2}$ University of Žilina, Žilina, Slovakia \\ ${ }^{3}$ Institute of Physics of Materials, Brno, Czech Republic
}

\begin{abstract}
Direct Metal Laser Sintering (DMLS) is one of the additive manufacturing technologies involving a metal powder bed and a laser source to obtain components of complex geometry. The fatigue life of an as-built DMLS part is negatively influenced by the rough surface compared to a machined surface. Since most DMLS components are expected to have also stress concentrations, here the combined effect on fatigue of a as-built surface and a geometrical notch in as-built DMLS Ti6Al4V was investigated using a new test methodology. Miniature specimens having three different directions with respect to build direction were fabricated and tested determining the directional fatigue notch sensitivity of as-built DMLS Ti6A14V Optical inspection of notch surface quality and roughness measurements demonstrated a ranking of the directional notches that inversely correlated to the measured fatigue performance. The link among the curved surface orientation, the peculiar layer-wise additive manufacturing fabrication and the fatigue notch sensitivity is clarified.
\end{abstract}

\section{Introduction}

Direct Metal Laser Sintering (DMLS) is one of the additive manufacturing technologies suitable for the rapid processing of a metal powder bed to obtain components of complex geometry with minimal material and energy wastes, [1]. A range of important metal alloys, such as Ti alloys, Ni-based super alloys, $\mathrm{Cr}-\mathrm{Co}$ alloys and Al-Si alloys, can be processed with a laser providing the concentrated thermal energy source that selectively fuses thin regions of a powder bed that quickly solidify. The process is repeated layer after layer until the entire part is built.

Whereas the effect of defects in materials prepared by DMLS has been widely discussed and the setting of optimal DMLS process parameters can eliminate their formation in a significant way, the problem of as-built surface roughness needs further investigation because in a part with a complex geometry, most of the surface area will neither be accessible for machining nor polishing and will remain in the as-built condition. When used in service the fatigue life of the as-built DMLS part is negatively influenced by the surface roughness typical of the process. The magnitude of the as-built surface roughness is dependent on melting energy, powder size distribution, the thickness of each built layer, [2].

The surface topography consists of multiple sharp radii of curvature that act as micro notches and lead to local stress concentrations, [3]. Therefore, fatigue crack initiation in as-built surface occurs at considerably lower stresses compared to a conventional machined surface.

The importance of the DMLS technology, the fact that Ti6Al4V is most commonly used material in the aerospace industry for its excellent overall properties and the lack of material allowables needed for part design have motivated the investigation of the fatigue properties of selective laser melted Ti6Al4V using smooth specimens with as-built surfaces, [4-9].

However, few metal additively manufactured components are expected to have simple geometries without any corners or radii that would act as stress concentrations. Therefore, the combined effect on fatigue of a rough as-built surface and a geometrical notch needs to be clarified to enable structural part qualification for service, [10]. Kahlin et al. originally reported the fatigue behavior of DMLS Ti6Al4V with as-built surfaces and quantified the notch fatigue factor for as-built tensile bar with a circumferential notch. The surface roughness itself was found to contribute most significantly by comparison of the fatigue strength of as-built and machined smooth specimens. The notch fatigue factor for combination of a geometrical notch and the rough surface was also determined in [10].

The loading axis of the notched specimens used in [10] was parallel to the build direction. Understanding the influence of fabrication orientation, process parameters and surface curvature on the effective as-built quality and its impact on fatigue strength is a key topic to be further explored. 
This study focuses on the experimental investigation of the surface quality of as-built notches having different orientations with respect to the build direction and the resulting sensitivity of DMLS Ti6A14V to notches and fatigue. The approach introduced in [11] based on the use of directional miniature specimens is adopted to determine the combined effect of an as-built notch and its fabrication direction with respect to build direction on the notch fatigue response.

\section{Experimental details}

Details of the material and its fabrication process, the peculiar specimen geometry and fatigue test methodology used, and the characterization techniques of the as-built surface roughness are now provided.

\subsection{Material and specimen production}

The Ti6Al4V ELI alloy powder used here was characterized by spherical powder particles of predominant diameter range from 25 to $45 \mu \mathrm{m}$. Specimen production was performed with the DMLS system EOSINT M290 (EOS GmbH, Germany) working with a layer thickness of $60 \mu \mathrm{m}$, a max laser power of $400 \mathrm{~W}$ and a chamber temperature of $80^{\circ} \mathrm{C}$. The contour and hatch strategy was adopted to melt the powder layerby layer. Hatching direction was rotated from one layer to the next of a prescribed angle.

After fabrication, the following stress relief heat treatment was applied: heating up to $740{ }^{\circ} \mathrm{C}$, exposure for $2 \mathrm{~h}$ and slow cooling to $540{ }^{\circ} \mathrm{C}$ all in a vacuum furnace followed by rapid cooling in Argon. The resulting quite isotropic structure was investigated elsewhere, [12]. The tensile properties of the heat treated DMLS Ti6A14V were also reported elsewhere, [13].

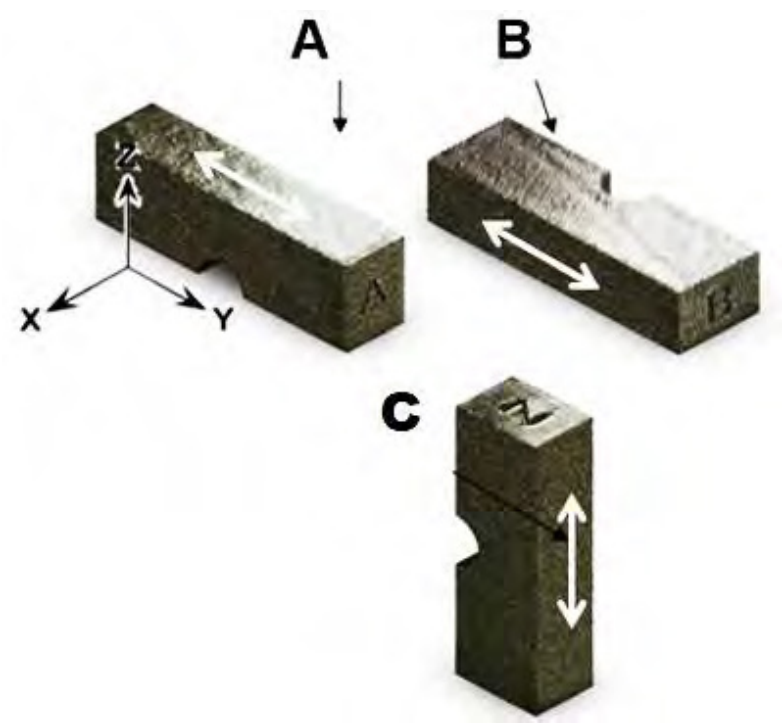

Fig. 1. Mini specimen orientations with respect to build direction. White arrow defines the applied stress direction.

A special miniature prismatic specimen geometry introduced in [14] was adopted to investigate the directional and geometrical notch effect on fatigue. The
Fig. 1 shows the three different specimen directions with respect to build direction and their denomination. The size of the nominal section is $5 \times 5 \mathrm{~mm}^{2}$ and the length of the specimen is $22 \mathrm{~mm}$. The prismatic specimen geometry is characterized by the presence of a semicircular notch of $2 \mathrm{~mm}$ radius. The theoretical stress concentration factor was quantified in $\mathrm{K}_{\mathrm{t}}=1.63$ using the finite element method. This is a mild notch effect but a sharp notch effect is reported elsewhere, [15].

Since the specimens can be oriented as desired with respect to the build direction, the anisotropic fatigue response can be readily investigated. Three sets of such specimens for a total of about 80 specimens in a single build were readily and cheaply produced. All the surfaces were left in the as-built condition to maximize the roughness effect on fatigue.

\subsection{Surface roughness characterization}

The optical characterization of the as-built notch quality was performed on polished and non-etched specimens with a light microscope Zeiss Neophot 32. Details of the notch root irregularities and initiated cracks were observed on etched ( $10 \% \mathrm{HF}$ for $10 \mathrm{~s})$ specimens.

The as-built roughness of the notch root on the length $\sim 2 \mathrm{~mm}$ was measured on a Mitutoyo SJ 210 tester. The roughness parameters evaluated were $\mathrm{R}_{\mathrm{a}}$ (arithmetical mean roughness value) and $R_{z}$ (mean roughness depth), which are the most often used for roughness characterization in industry. The focus was the determination of the link between build direction of the notch and local roughness.

\subsection{Fatigue test methodology}

The three sets of mini specimens oriented in three different directions with respect to the build direction were tested in fatigue using a plane bending machine under a load ratio $\mathrm{R}=0$ with run-out fixed at $2 \times 10^{6}$ cycles.

The validation of the test methodology used here, [14], compared the plane bending test results of as-built Type $\mathrm{C}$ specimens and the rotating bending test results of as-built 6-mm-diameter specimens with longitudinal axis parallel to the build direction. The two sets of specimens were fabricated in DMLS Ti6Al4V. Since the two independent fatigue curves showed coherent trends, this new test methodology can be conveniently used,.

Since the miniature specimen geometry is characterized by a flat surface and a semicircular notch on opposite faces, Fig. 1, the tensile cyclic stress was applied either on the flat surface, see white arrows in Fig. 1, to obtain the un-notched reference fatigue behavior or on the opposite notch root to obtain the notch fatigue behavior as schematically shown in Fig. 2.

Thus the three set of specimens are used to determine the directional un-notched and notched fatigue performance of the as-built DMLS Ti6A14V, [8]. 


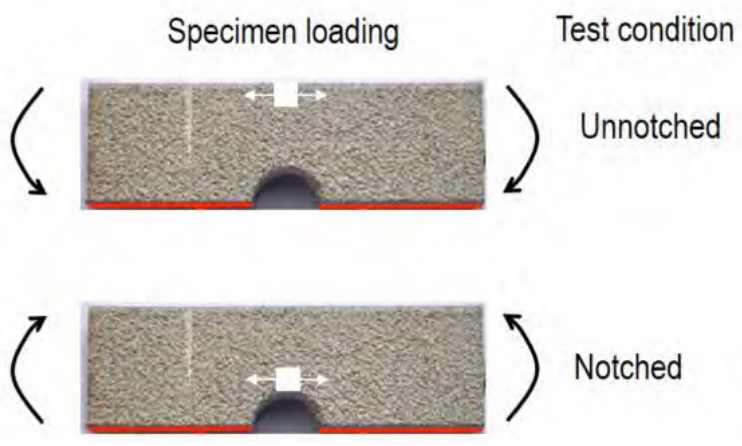

Fig. 2. Alternative cyclic bending loadings with $\mathrm{R}=0$ of the specimens and different conditions investigated.

\section{Results and discussion}

This section presents initially the notched fatigue behavior of the DMLS Ti6Al4V obtained with the directional mini specimens followed by the as-built surface characterization and roughness measurements at the notch root. Observations of fatigue crack initiation show the interaction of crack and the local surface conditions. A technology-based explanation of the directional notch fatigue behavior is finally introduced.

\subsection{Notch fatigue behavior of DMLS Ti6AI4V}

\subsubsection{As-built notch fatigue behavior}

Fig. 3 shows the combined directional and notch fatigue effect obtained in the three sets of the present mildly notched mini specimens. The maximum nominal stress of the bending load cycle with $\mathrm{R}=0$ is used as independent variable. Three different and well defined trends are determined for the different directions of fabrication.

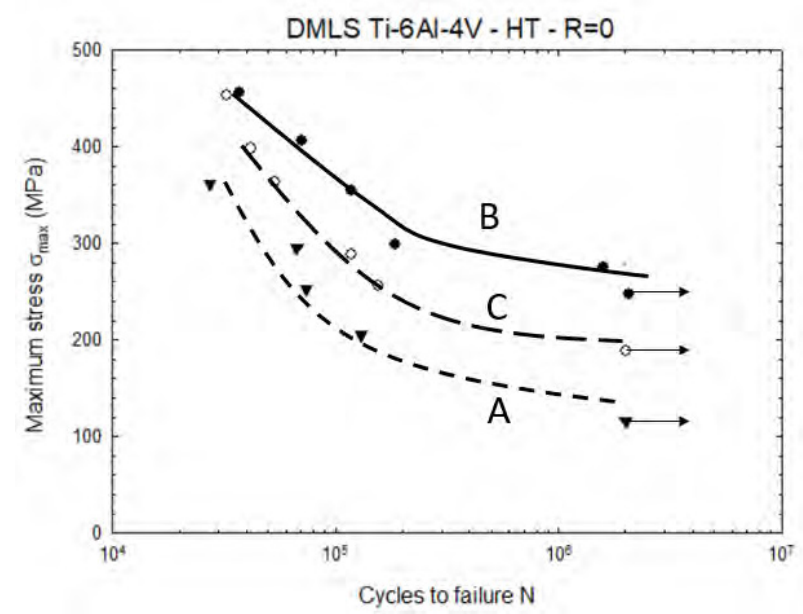

Fig. 3. Directional notch fatigue behavior for the same stress concentration factor.

Considering Fig. 1, the Type B specimen shows the best fatigue performance while the Type A specimen the worst fatigue performance. Type $\mathrm{C}$ specimens determine an intermediate behavior. The directional fatigue behavior of un-notched DMLS Ti6Al4V after the same stress relieving treatment used here was determined with the three sets of mini specimens and presented elsewhere, [8]. For the purpose of this study the data could be grouped in a unique scatter band with a mean representative high cycle fatigue strength of about 360 $\mathrm{MPa}$ at $2 \times 10^{6}$ cycles. This value will be used to in the estimation of the notch fatigue factor in a subsequent section.

\subsubsection{Notch fatigue factor}

The classical definition of notch fatigue factor $\mathrm{K}_{\mathrm{f}}$ is the ratio of the un-notched and of the notch high cycle fatigue strengths, see [16]. Typically, the surface finish considered depends on conventional fabrication processes (i.e. machining) and $\mathrm{K}_{\mathrm{f}}$ quantifies only the geometrical notch effect on fatigue.

Here according to this approach, the un-notched and notched fatigue strength data to be examined are for asbuilt surfaces. Therefore, considering the reference unnotched fatigue strength of $360 \mathrm{MPa},[11]$, three distinct $\mathrm{K}_{\mathrm{f}}$ values (i.e. one per specimen direction) are estimated from the present experiments on DMLS Ti6Al4V, namely $\mathrm{K}_{\mathrm{f}, \mathrm{A}}=2.6, \mathrm{~K}_{\mathrm{f}, \mathrm{B}}=1.4$ and $\mathrm{K}_{\mathrm{f}, \mathrm{C}}=1.6$ where the second subscript defines the notch direction with respect to build direction.

Since the geometrical stress concentration factor of the notched mini specimens is $\mathrm{K}_{\mathrm{t}}=1.63$, the following more general result is reached for DMLS Ti6Al4V:

$$
\mathrm{K}_{\mathrm{f}, \mathrm{A}}>\mathrm{K}_{\mathrm{t}} ; \quad \mathrm{K}_{\mathrm{f}, \mathrm{B}}<\mathrm{K}_{\mathrm{t}} \quad \mathrm{K}_{\mathrm{f}, \mathrm{C}} \approx \mathrm{K}_{\mathrm{t}} .
$$

The as-built notch effect in fatigue is apparently directional due to a technology-dependent effect superposed to the classical geometrical notch effect.

These findings are possibly the first of this kind and therefore they are not readily validated. Nonetheless, recent valuable fatigue data of [10] for with as-built unnotched and notched specimens made of DMLS Ti6Al4V defined a notch fatigue factor $\mathrm{K}_{\mathrm{f} .}=2.4$ for a sharper notch geometry (i.e. $\mathrm{K}_{\mathrm{t}}=2.5$ ) and a different loading condition (i.e. cyclic tension, $\mathrm{R}=0.1$ ). Since the specimen axis of [10] was parallel to the build direction, as here is the case for Type $\mathrm{C}$ specimens, the result of [10] support the previous result that $\mathrm{K}_{\mathrm{f}, \mathrm{C}} \approx \mathrm{K}_{\mathrm{t}}$. Further investigations on different powder bed fused metals are underway.

\subsection{As-built notch surface quality}

This section presents the characterization of the notch surfaces of the three specimen types of Fig. 1 using magnified optical microscopy and roughness measurements.

Fig. 4 to 6 show the magnified view of the round notches of the three specimen types along with the build direction. The images confirm the strong dependence of the surface quality in terms of roughness and adherence 
to the theoretical semi-circular geometry on the fabrication parameters.

Especially important for discussing the notch fatigue test results, it is the roughness of the notch root where the stress concentration is localized and crack initiation occurs. Inspections of Fig. 4 to 6 show qualitatively that the surface roughness of the notch is the lowest for the Type B specimen and the highest for Type A specimen. Type $\mathrm{C}$ specimen shows a roughness that depends on the local surface orientation with respect to the build direction.

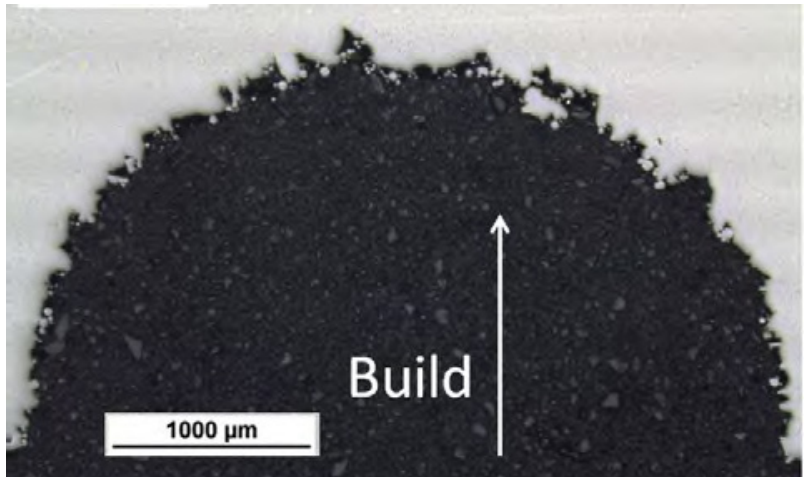

Fig. 4. As-built surface roughness of Type A round notch.

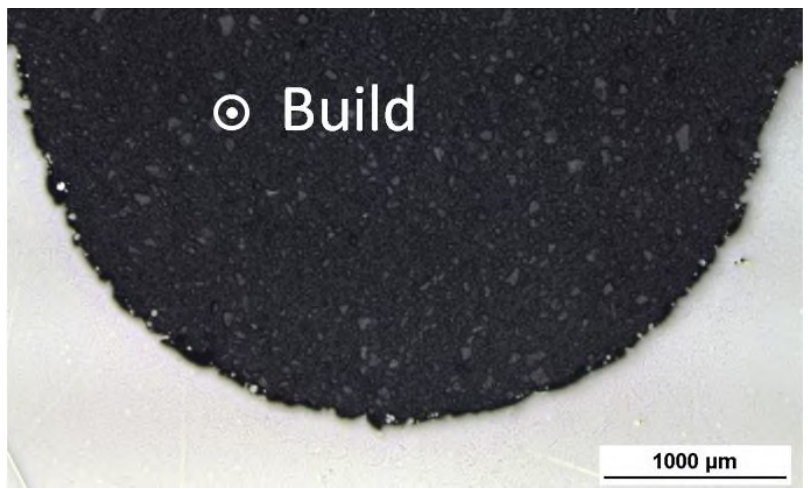

Fig. 5. As-built surface roughness of Type B round notch (note that build direction is perpendicular to page).

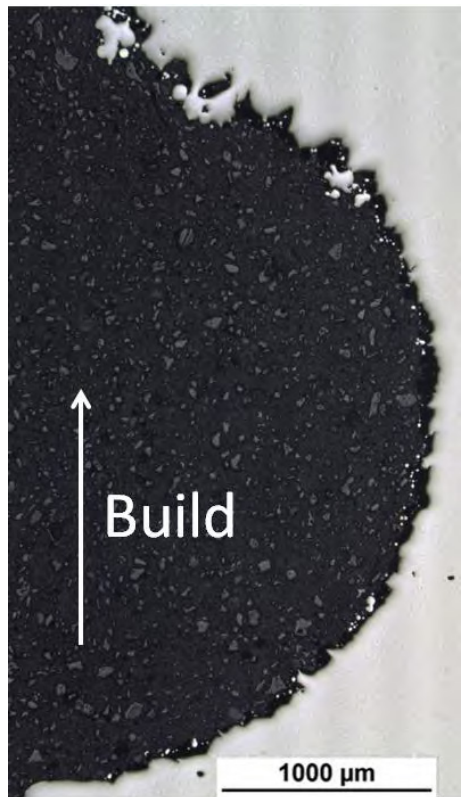

Fig. 6. As-built surface roughness of Type $\mathrm{C}$ round notch.
Roughness measurements of parameters $R_{a}$ and $R_{z}$ were performed at the root of each type of notch and are presented in Table 1. Since the surfaces were curved, the measurement length was shorter than the length recommended by the standard.

Table 1. Notch root roughness vs fatigue specimen type.

\begin{tabular}{|c|c|c|c|}
\hline Parameter & Type A & Type B & Type C \\
\hline $\begin{array}{c}\mathrm{R}_{\mathrm{a}} \\
{[\mu \mathrm{m}]}\end{array}$ & $20.506 \pm 3.6$ & $8.531 \pm 0.8$ & $10.105 \pm 0.6$ \\
\hline $\begin{array}{c}\mathrm{R}_{\mathrm{z}} \\
{[\mu \mathrm{m}]}\end{array}$ & $107.599 \pm 21.596$ & $44.835 \pm 3.3$ & $54.599 \pm 5.9$ \\
\hline
\end{tabular}

The average roughness measures $R_{a}$ and $R_{z}$ are however coherent with the visual inspection of Fig. 4 to 6 . Furthermore, the data of Tab. 1 demonstrate that the surface roughness of the notch root of the three types of specimens having the same nominal semicircular geometry inversely correlates with the experimental fatigue ranking of Fig. 3. Type B has the lowest roughness and the highest notch fatigue strength while Type A has the highest roughness and the lowest notch fatigue strength. Type $\mathrm{C}$ has an intermediate roughness and a intermediate notch fatigue strength.

\subsection{Fatigue crack initiation at notch root}

The previous views of the notches gave an overall view of the surface quality in dependence with surface orientation. Now etched details of the notch roots for the different specimens of Fig. 7 to 9 show the interaction of local roughness and material structure and the fatigue crack mechanisms.

Fig. 7 shows the high roughness of the surface of a Type A specimen with evidence of partly melted particles and dross formation due to the down-skin orientation of this type notch. Sharp re-entrant notches are typically observed favoring multiple crack initiation observed. The influence of down-skin notch quality vs up-skin notch quality on fatigue behavior of DMLS Ti6Al4V has been discussed recently in [8]. Down-skin notches showed the worst fatigue performance.

Fig. 8 shows the notch root contour of Type B specimens which is much more regular than the previous Fig. 7. The shape is locally a circular arc and the stress concentration is expected to be well described by the $\mathrm{K}_{\mathrm{t}}$ as in the case of a machined surface. The etched structure reveals the contour path and the equiaxed structure of the hatched internal area.

Finally, Fig. 9 shows the notch root contour of Type $\mathrm{C}$ specimens with a fatigue crack at the center. Globally, the roughness is intermediate compared to the previous Figs. 7 and 8. Interestingly, the upper notch arc shows an increasing roughness compared to the lower part. This is due to the influence of surface orientation with respect to the build direction. 


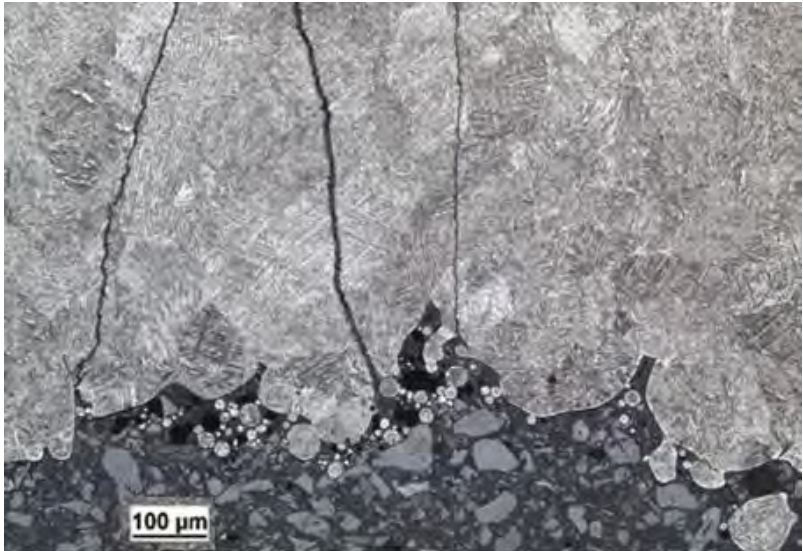

Fig. 7. Magnified Type A notch root and fatigue cracking

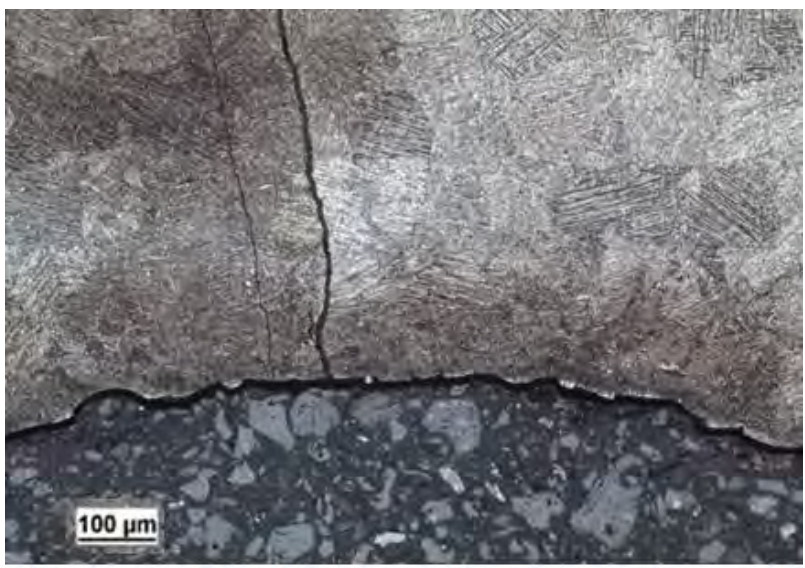

Fig. 8. Magnified Type B notch root and fatigue cracking

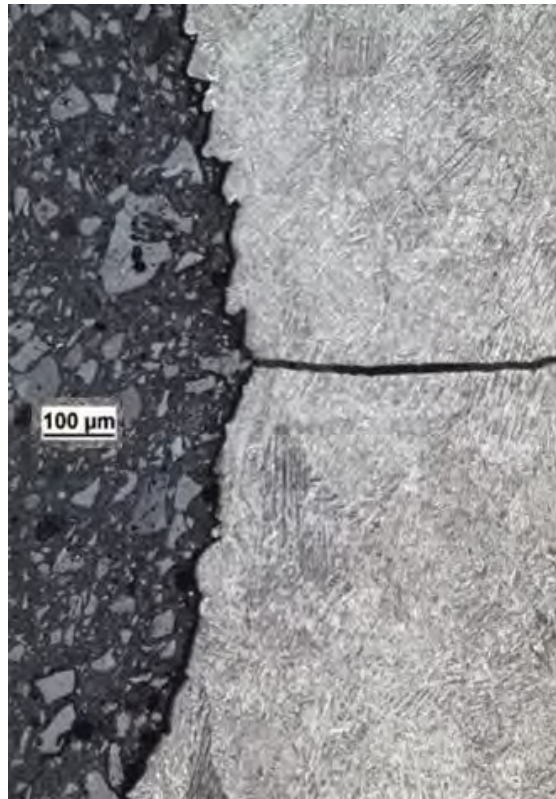

Fig. 9. Magnified Type $\mathrm{C}$ notch root and fatigue cracking

\subsection{Directional layer-wise notch generation}

The layer-by-layer notch generation is now schematically introduced in Fig. 10 to 12 to provide a link between the technology-dependent contribution to notch roughness and the directional fatigue strength of the three specimen types.
The notch root of Type A specimens, shown in Fig. 10 , is affected very significantly by the step-wise approximation of circular geometry. The layer thickness is also expected to give an increasing negative contribution in fatigue. On the other hand, the notch root geometry of Type B specimens is not affected by the layer-wise fabrication, Fig. 11. The contour and hatch strategy typically used defines accurately the circular notch geometry by contouring. Finally, the notch root of Type C specimens is globally affected by the step-wise approximation of circular geometry but the notch root is affected the least, Fig. 12. In this case, however, the stress concentration is however acting perpendicular to the layers, which is normally considered the weakest direction.

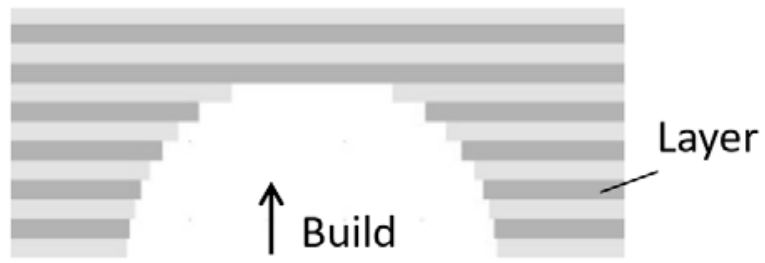

Fig. 10. Layer-wise generation of the Type A round notch.

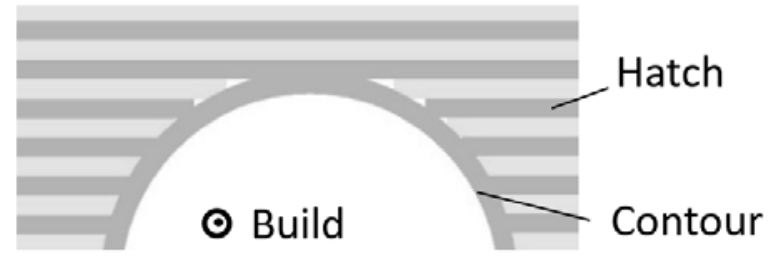

Fig. 11. Contour and hatch strategy used for obtaining the Type $\mathrm{B}$ round notch (note that build direction is perpendicular to page).

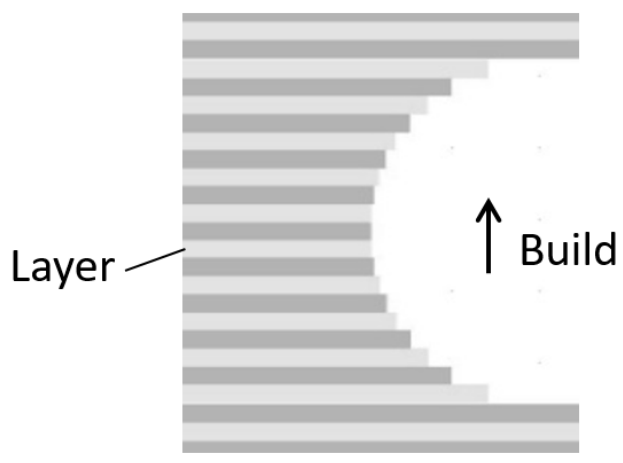

Fig. 12. Layer-wise generation of Type $C$ round notch.

The schemes of Fig. 10 to 12 provide a link between curved surface fabrication direction and fabrication parameters and therefore the technological-dependent negative contribution to the geometrical notch effect quantified in Fig. 3.

\section{Conclusions}

This study was focused on the experimental investigation of fatigue notch sensitivity of as-built DMLS Ti6Al4V the presence of different orientations with respect to the 
build direction using a test methodology recently introduced, [14]. The main conclusions are the following:

- The test methodology using miniature fatigue specimens efficiently provided original insight into the dependence of the fatigue behavior on the directionality of the notch fabrication.

- Of the three directional specimens investigated and shown in Fig. 1, Type A showed the worst notch fatigue performance, Type $\mathrm{B}$ the best and Type $\mathrm{C}$ an intermediate notch fatigue performance.

- Optical inspection of the notch surface quality and roughness measurements demonstrated a ranking of the directional notches that inversely correlated to the measured fatigue performance.

- Three directional notch fatigue factors $K_{f, A}, K_{f, B}, K_{f, C}$ were originally estimated from the present data for the same $\mathrm{K}_{\mathrm{t}}$ demonstrating a technological contribution in addition to the geometrical effect.

- A recent notch fatigue factor from the literature supported the present estimate for the same material and notch orientation.

- The link among the curved surface orientation, the peculiar layer-wise additive manufacturing fabrication and the fatigue notch sensitivity is schematically defined.

Part of the research was supported by the project Slovak VEGA grant No. 1/0685/2015. Specimen fabrication by the technological partner BEAM-IT, Fornovo Taro (Italy) is gratefully acknowledged.

\section{References}

1. W.E. Frazier, J. Mater. Eng. Perform., 23 (2014).

2. J. Kranz, D. Herzog, C. Emmelmann, J Laser Appl 27 (2015).

3. K.S. Chan, Surf Topogr Metrol Prop 3 (2015).

4. S. Leuders, M. Thöne, A. Riemer, T. Niendorf, T. Tröster, H. Richard, Int J Fatigue 48 (2013).

5. E. Wycisk, A. Solbach, S. Siddique, D. Herzog, F. Walther, C. Emmelmann, Phys. Proc, 56, (2014).

6. P. Edwards M. Ramulu, Materials Science and Engineering: A, 598, (2014)

7. T. M. Mower, M. J. Long, Materials Science and Engineering, A651 (2016)

8. Greitemeier D, Palm F, Syassen F, Melz T. Int J Fatigue 94 (2017)

9. P. Li, D.H. Warner, A. Fatemi, N. Phan, Int J Fatigue, 85 (2016)

10. M. Kahlin, H. Ansell, J.J. Moverare, Int J Fatigue 101 (2017)

11. G. Nicoletto, Int J Fatigue, 106 (2018)

12. R. Konečná,, G. Nicoletto, A. Bača, L. Kunz, Materials Science Forum, 891 (2017).

13. R. Konečná, G. Nicoletto, S. Fintová, M. Frkáň, Structural Integrity Procedia 7 (2017)

14. G. Nicoletto, Int J Fatigue, 94 (2016)
15. M. Frkáň, G. Nicoletto, R. Konečná, Procs. XIX ICMFM, Porto, Portugal (2018) (in preparation)

16. R. Juvinall, K.M. Marshek, Fundamentals of Machine Component Design, New York: John Wiley, (2012) 